\title{
Current Situation and Countermeasures of WeChat Application in E-government Affairs
}

\author{
Liyuan Shang ${ }^{1}$, Zhenting $\mathrm{Lu}^{2}$, Xiang Liu ${ }^{3}$ \\ 1 Department of Economics and Management, Jiangxi Technical College of Manufacturing, \\ JiangXi Nanchang, 330095 \\ 2 Jiangxi Industry Polytechnic College, Electronic and Information Engineering Branch, , JiangXi \\ Nanchang, 330095 \\ 3 Jiangxi Technical College of Manufacturing, Department of Information Engineering, JiangXi \\ Nanchang, 330095
}

Keywords: Government WeChat; Public Platform; Governmental Service; E-government Affairs

\begin{abstract}
The combination of WeChat and government affairs, being labeled by "Shake", has gradually become a major platform to reflect the social conditions and public opinions. Due to the unique advantages of immediacy, interactivity, privacy and economy, WeChat springs up in many social media networks, and plays an increasingly important role in the distribution and publication of government affairs. With the further expansion of government WeChat teams, the government shall first know about how to combine with the practical works of the government, play the "communication", "convenience for the people" and "administration" values of government WeChat and build the service-oriented government.
\end{abstract}

\section{Introduction}

With the development of message network technology and communication technology, all aspects of people's lives have been dramatically changed. As a new thing in the information age, E-government Affairs is the organic combination of electronic science and technology and government agencies, and it has become a new way of government offices. E-government Affairs uses the computers and network information technology to achieve the purpose of the government administration, effectively promote the development, utilization and sharing of government information resources, improve the efficiency of government administration and decision-making levels of the government officials, strengthen the government regulation, improve the transparency of law enforcement, improve the quality of public services and governmental services, help the government and people effectively carry out direct communication, and promote the development of national economy and social message.

\section{Current Situation of WeChat Application in E-government Affairs}

This paper mainly analyzes from the account features, release contents, strengths and weaknesses of government WeChat.

Account Features. Geographical distribution: In terms of geographical distribution, Zhejiang, Shandong and Guangdong have the largest number in opening the government WeChat accounts. The government WeChat awareness of southern provinces is stronger than the Northern provinces. 58 accounts belong to the southern provinces, whereas 37 accounts belong to the Northern provinces. Therefore, government WeChat shows the tendency of more in the east and less in the west, more in the south and less in the north, and the difference is relatively significant.

Profile photo: Through observation, all government WeChat account photo can be divided into the following categories: cartoon, department signs, office buildings and character photos.

Verification: This study searches by the keyword of "government" and screens 95 valid accounts. Verification includes three forms: Sina microblogging verification, Tencent 
microblogging verification and $\mathrm{WeChat}$ verification. As long as any accounts are verified, the lower left of the picture will appear the "V" sign, and thus increase the credibility of the account.

The primary government WeChat accounts and low-level government WeChat accounts: The primary government $\mathrm{WeChat}$ accounts refers to the accounts opened by the primary governments that can represent the people's government, whereas low-level government WeChat accounts refers to WeChat accounts opened by a specific government departments or government agencies.

Analysis of Release Contents. First greetings: After becoming friends with the government WeChat account, some accounts will immediately release a greeting message. Greetings can increase opportunities for interaction with users. Among the followed 95 accounts, only three accounts did not send greetings, accounting for $3 \%$ of the total. First greetings consist of simple welcome and navigation guide welcome, $64 \%$ of the accounts send simple welcome, and only $33 \%$ of WeChat accounts send navigation guide welcome. Navigation guide welcome is more directional, telling users how to reply to get targeted answers in details. It not only brings convenience to users, but also reduces the delay of labor response.

Release form and release contents: Release form includes plain text messages, image-text message, video message and voice message. Observations show that 75 accounts release messages by using the image-text form, 1 account uses the video form; 17 accounts use plain text form; 8 accounts use the voice form.

One advantage of WeChat release is its voice function. With voice messages, it is easier to narrow the distance between government and users, and it is also an obvious advantage of WeChat compared with MicroBlog. The observed data show that the government seldom uses the voice function. The plain text and image-text messages account a higher proportion.

Analysis of Strengths and Weaknesses. Strengths: Firstly, WeChat push has a higher system priority (will be pushed to the notification bar, and the voice prompt is defaulted), so that users may receive messages at first time.

The platform is more stable. Since it is a social relation network, the relationship between users is closer, and the solid relationship may ensure the quantity and quality of the users.

Customer relationship is more equal. Due to point-to-point communication and two-way interaction, the relationship between WeChat users is more equal, and their exchanges are smoother. The smart reply function of WeChat public platform also facilitates to narrow the distance between enterprises and users.

Message is selected and the positioning is accurate. WeChat message push can be completed instantaneously and synchronously, there is no time difference for all people to receive messages, which also guarantee the timely transmission of messages, avoid the misleading, processing and loss of middle links.

Weaknesses: Keyword automatic reply feature is a major advantageous positioning of the WeChat public platform product, that is to say, WeChat is a chat robot. To take WeChat public platform as the chat robot, thesaurus is the biggest problem. Even without considering the thesaurus, WeChat also does not provide a more complete solution to multiple keyword priority issue.

Public platform actively push messages, but users receive message within a limited scope. When the messages exceed this limit, users will certainly cancel some other WeChat pushers, the first entrants have their own advantages, and they can quickly accumulate users, causing barriers to users.

Opportunities: In the web2.0 era, social networking has become an increasingly apparent trend. More and more people like to use WeChat to follow their friends and family members. Based on mobile phone and modern message technology, the mobile internet will become the largest message push service platform, the largest new media and largest potential marketing channel in the world. 
Threats: The users may unsubscribe at any time. As a direct push to smart phones, "spam" and "quality message" are available for the audience. Once they are dissatisfied with the time (for example, push at work or busy periods) or contents, users may be reduced and lost.

Above all, the strengths of WeChat public platform application in E-government Affairs outweigh the weaknesses, and opportunities outweigh the threats.

\section{Countermeasures of WeChat Public Platform Application in E-government Affairs}

In summary, despite great progress made in the Chinese government WeChat operation, there are still too many problems, and there is still much space for improvements. The capability of fully application of the government WeChat platform is a specific embodiment of the government administration capability in the social media era. According to quantitative and qualitative analysis, this paper puts forward the following countermeasures to the Chinese government WeChat management.

Recommendations on Regional Public Account Opening. At present, the government WeChat opening regions are mainly in southeast provinces, and regional differences are significant. Among the searched top 100 accounts (5 non-related accounts), 95 other accounts are sorted. Among the 95 valid government WeChat accounts, 61 accounts come from the eastern region; 13 government $\mathrm{WeChat}$ accounts come from the central region; $21 \mathrm{WeChat}$ accounts come from the western region. The central, western and northern regions shall actively follow up, and open the government WeChat as soon as possible.

Recommendations on Profile Photos and Greetings. Humanized government WeChat profile photo facilitates to narrow the distance between the Government and the audience. As a widely used interactive platform, WeChat should be an effective tool to improve communication between the government and the public, and the formal profile photo will alienate the users. First greetings should also send messages in a considerate manner. Regardless of the digital navigation or text search functions, it allows users to understand the interaction method at a glance, in order to quickly get the needed messages, and reduce the workload of manual reply to some extent.

Besides, voice message greetings may also be frequently used, which is a major advantage to distinguish from WeChat public platform, and it can also accepted by the audience.

Recommendations on Release Language and Communication Language Use. First of all, the language should not be too "professional". The document formulation shall be professional. However, as an interactive message platform, the language should not be too professional.

Secondly, it should not alienate the public. The messages should be released in a popular and accurate manner, so that the public can easily understand. It is hard for the audience to accept the released messages with excessive formal language.

Finally, message notification shall be standardized and accurate. The government should administer through message release, accept the supervision and judgment from the public. If the messages are obscured, the public supervision will become impossible.

Enrich Government WeChat Release Form and Improve WeChat Release Frequency. Online instant voice transmission function is a big advantage for WeChat to penetrate into the public cultural life as a new social media. However, government WeChat is not fully applied to message release and interaction. The study found that the voice message released by the government WeChat accounts is less than 5\%. In addition, video and audio streaming media have been widely used in social networking. The government WeChat should take full advantage of these features, and stimulate the interests of users and improve the user attention. Besides, in some specific services, voice message is more necessary than text and image message. For example, when Transportation Bureau releases the instant road messages, the audiences are drivers, so it is obvious that sending text messages at this time may affect driving safety, and 
voice messages may bring convenience to users. In addition, some government WeChat should also further improve the release frequency and stability of release interval.

\section{Conclusions}

As a key product of digital technological development, WeChat has become one of the tools of close human relationship at the current stage, causing more and more prominent influences on lives, study and works. Especially in the current circumstances, the number of Tencent WeChat users has developed to 300 million, which only spent for less than two years. Therefore, WeChat is the fastest growing instant messaging tool up to now, not only impacting the traditional media, but also wining favor from the government. This paper systematically analyzes the WeChat public platform application in E-government Affairs, studies the government affairs messages released by the government, and describes the strengths, weaknesses, opportunities, threats and future development trends of WeChat public platform application in E-government Affairs.

\section{References}

[1] Zhang Erxu. Viral Marketing Analysis of WeChat Promotion [J]. Marketing Planning, 2012 (6).

[2] Public Security Affairs WeChat Is Coming, Please "Shake". 2013-01-15.

[3] Guidelines to Government Affairs Microblogging Registration Verification. 2013-12-09.

[4] @Pingan Zhaoqing Launches "Government WeChat" Following the Microblogging.2012-10-19.

[5] WeChat User Manual. 2013-12-09.

[6] Chinese Academy of Governance E-government Affairs Research Center, Assessment Report for Chinese Government Affairs Microblogging in 2013.

[7] Wu Aimin, Foreign E-government Affairs, Taiyuan: Shanxi People's Publishing House. 2013: 4-5.

[8] Zhao Guojun. E-government Affairs Tutorials, Beijing: China Renmin University Press, 2013, 6-7.

[9] Zhang Ruixin, E-government and E-government Affairs, Beijing: China Renmin University Press, 2012, 110-112.

[10] Wang Ping, Innovation of Urban Information and Governance Model, Doctoral Thesis, East China Normal University, 2012.

[11] Liu Chunnian, Research on E-government Affairs Development Mechanism, Doctoral Thesis: Wuhan University, 2013.

[12] Kaifeng Yang. Neo institutionalism and E-government Affairs: Beyond Jane Fountain. Social Science Computer Review. 2013(21).

[13]Stuart Bretsehneider. message technology, E-government Affairs, and institutional change. Public Administration Review. 2012(63). 